fetal serum AFP that enters the amniotic fluid in the presence of a normal conceptus.

Our results and those of Ruoslahti and colleagues emphasise that the AFP con Abinding assay on amniotic fluid has particular value in detecting the presence of communicating fetal abnormalities during the period when amniocenteses are usually performed, between the 14th and 18th weeks, in cases where the elevations of amniotic fluid AFP are equivocal or when gestational aging is uncertain.

Philip C KeLleheR Sir William Dunn School of Pathology, Oxford OX1 3RF

Department of Medicine,

University of Bristol,

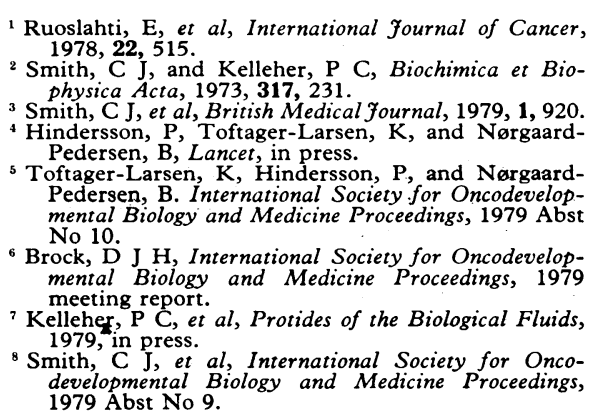

CAROL J P SMITH

\section{Endotoxins and gastroduodenal} ulceration after burns

SIR,-Your leading article "Gastric and duodenal ulceration after burns" (1 September, p 512) exactly indicates that we still know little about how this occurs. In an attempt to identify some of the mediators involved in the pathogenetic mechanism of these multiple erosions, we would like to suggest a possible role of endotoxins.

In fact, experimental investigations ${ }^{1}$ have demonstrated that endotoxins of intestinal origin enter the circulation because the intestinal wall is made more permeable by the release into the blood of a vasoactive substance from the burned skin. In addition, fatal endotoxaemia (detected by the Limulus endotoxin assay) developed in normal rabbits that received the venous blood from the burned area before it contained endotoxin; by contrast, absence of endotoxaemia and the survival of rabbits which did not harbour Gramnegative bacteria in the intestine was noted. Recent clinical observations ${ }^{2} 3$ have added credibility to the importance of endotoxaemia in burned patients. Since the myriad of biological activities of endotoxins includes also a direct effect on the gastric mucosal circulation, permeability, and morphology, ${ }^{4}$ we-like others ${ }^{5}$-think that significant mucosal ischaemia can occur and may represent a mechanism in the development of gastric erosions in endotoxaemia after large burns.

University Department of Medical

D Fumarola Microbiology,
70124 Bari, Italy

${ }^{1}$ Cuevas, P, et al, Gynecology and Obstetrics, 1974, 138, 726.
Harris, N S, and Feinstein, R, fournal of Trauma, $1977,17,714$.

Jones, R J, and Roe, E A, fournal of Hygiene (Cambridge), $1979,83,151$.

- Cheung, L Y, et al, fournal of Surgical Research, 1975, 18, 417 .

Cheung, L Y, Reese, R S, and Moody, F G, Surgery,
$1976,79,564$.
High-carbohydrate diets and insulin-dependent diabetics

SIR,-Dr N M O'Mullane suggests (29 September, p 796) that the improved diabetic control we observed in insulin-requiring diabetics on high-carbohydrate diets (1 September, p 523) could be explained by a difference in dietary fibre between the experimental diet and the standard low-carbohydrate comparison diets.

Unfortunately Dr O'Mullane did not refer back to the earlier publication (30 June, p 1753) in which we presented results of a similar dietary comparison in maturity-onset patients and where details of the diets were provided. The high-carbohydrate diets did indeed contain appreciably more dietary fibre than the low-carbohydrate diet. We agree that our own published work thus far provides no conclusive data concerning which aspect of the high-carbohydrate diets is responsible for the improved diabetic control. We did, however, quote various studies which suggest that the reduced glucose baseline on a high-carbohydrate diet may be a consequence of digestible carbohydrate. Ongoing dietary studies in Oxford aim to determine the optimal proportion and nature of carbohydrate in the diabetic dietary prescription.

University Department of Social

and Community Medicine, Oxford OX1 $3 Q N$

\section{Treatment of adder bites}

SIR,-I welcome the letter from Dr Margaret T McKiddie (6 October, p 865). Although antivenom is needed in only a minority of adder bites, the withholding of Zagreb antivenom may be hazardous when serious poisoning continues in hospital, especially hypotension.

Such cases are unpredictable and warrant careful observation of the patient-preferably in an intensive care unit. Children are usually bitten at weekends; and observation, even in teaching hospitals, may at that time be remiss. Unfortunately hospital doctors are often ignorant about the basic points of observation in adder-bite poisoning (especially observation for hypotension, electrocardiographic changes, and neutrophilia). This can have tragic results. ${ }^{1}$.

WHO Collaborative Centre for

Alistair ReID

the Control of Antivenoms,
Liverpool School of Tropical

Medicine,

Liverpool L3 5QA

${ }^{1}$ Reid, H A, British Medical fournal, 1976, 2, 153.

\section{Choosing a doctor in the USSR}

SIR,-There are difficulties in choosing, or even finding, a good personal doctor in the USSR, as everywhere else. I am surprised, however, that, with two pages at his disposal, Dr Michael Ryan (20 October, p 979) did not recall one interesting example of how this problem can be at least partly solved, which both of us saw on a visit to a large polyclinic at the tractor works in Kharkov in 1974.

On a long table in the entrance lobby lay about 10 ledgers, each bearing the name of a doctor. Workers wishing to see one simply chose the doctor they preferred, looked for a vacant appointment, and wrote their own names into the diary. There was no receptionist to organise it. Of course, some doctors were more popular than others and were full up a day or two ahead; other less popular doctors could be seen more quickly. The appointments were at intervals of 15 minutes.

It seemed a simple idea, refreshingly free from bureaucracy, which might easily be applied here. Perhaps Michael Ryan forgot; or perhaps he thought it irrelevant to his rather narrow specialty in negative medical Kremlinology.

Glyncorrwg Health Centre,

JULIAN TUdOR HART Glamorgan SA13 3BL

\section{Withdrawal of cyanocobalamin}

SIR,-Professor D M Matthews and Dr J C Linnell (1 September, p 533) make valuable distinctions between the cobalamins. Not only is hydroxocobalamin at least as effective therapeutically in all circumstances as cyanocobalamin, but it is also safer in occasional patients with tobacco amblyopia, Leber's optic atrophy, and tropical polyneuropathy. In addition, the basic NHS cost quoted by Glaxo is the same for both.

Surely there is a very strong case for asking for the complete withdrawal of cyanocobalamin from the market. Eastern General Hospital,
Edinburgh EH6 7LN

N McD Davidson

***Dr Davidson sent a copy of his letter to Dr Goodspeed, medical adviser to Glaxo,

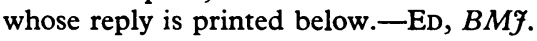

SIR,-Perusal of the data sheets for Cytamen (cyanocobalamin) and Neo-Cytamen (hydroxocobalamin) will confirm that the latter has indications not covered by the former. The data sheets for all products are readily available to all practitioners and none of the three conditions referred to by $\mathrm{Dr}$ Davidson has ever appeared in a data sheet for Cytamen.

Both products may be used in the treatment of pernicious anaemia, and practitioners may exercise their choice on the basis of their knowledge and experience. Unhappily, some will include in their experience a patient who has shown an anaphylactic response to either product. At present, the practitioner is free to switch such a patient to the other product, and so far we have no reports of cross sensitivity.

Cytamen is a highly satisfactory agent for the treatment of pernicious anaemia. Why should it be withdrawn if some practitioners use it in conditions for which it never has been the treatment and when it will enable trouble-free treatment of patients for whom an alternative agent has become unsuitable?

A $\mathrm{H}$ Goodspeed Medical adviser

Glaxo Laboratories Limited,
Greenford, Middx UB6 OHE

\section{The concept of disease}

SIR,-In addition to the endless debate whether so-and-so is a disease, we read of diseases in their own right, diseases sui generis, disease entities, entities, specific entities, true entities, and real entities. And we may ask whether some entity is a disease, or some disease is an entity. That majority of doctors who-in the words of your leading 